\title{
Association of HLA-G 14bp INS/DEL Polymorphism with brain morphology in Schizophrenia
}

\author{
Ashwini Rajasekaran ${ }^{1,2^{*}}$, Venkataram Shivakumar ${ }^{2}$, Deepthi Venugopal ${ }^{1,2}$, Sunil V. Kalmady ${ }^{2}$, Anekal C. Amaresha ${ }^{2}$, \\ Mahavir Agarwal², Janardhanan C. Narayanaswamy², Ganesan Venkatasubramanian², Monojit Debnath¹ \\ From International Conference on Human Genetics and 39th Annual Meeting of the Indian Society of \\ Human Genetics (ISHG) \\ Ahmadabad, India. 23-25 January 2013
}

\section{Background}

Multiple lines of evidence have implicated dysregulated immune processes in the pathogenesis of schizophrenia. Being an important immuno-modulatory molecule, Human Leukocyte Antigen (HLA)-G plays a pivotal role in successful pregnancy. Altered expression of HLA-G due to environmental and genetic variations not only lead to pregnancy complications but also a range of immunopathologies, some of which are being considered to confer risk for schizophrenia. One of the polymorphic marker, 14bp insertion/deletion (INDEL) located within the 3'UTR region of the HLA-G locus in Chr.6p21.3 is associated with HLA-G expression and function. The current study is aimed at analysing the role of $14 \mathrm{bp}$ polymorphism and the impact of feto-maternal compatibility/incompatibility at this locus on the risk of schizophrenia. In addition, the effect of 14bp INDEL on brain structure alterations in schizophrenia patients was also investigated.

\section{Methods}

A total of 151 (male-75 and female-76) schizophrenia patients and 113 (male-68 and female-45) ethnicity matched healthy controls $(\mathrm{HC})$ were considered. In addition, mothers of 64 schizophrenia patients were also recruited. Genotyping of 14bp INDEL was determined by PCR. Structural brain images were acquired using a 3Tesla MRI in $108 \mathrm{HC}$ and 76 schizophrenia patients. Voxel-Based Morphometry toolbox in SPM8 was utilized for brain imaging analysis using bilateral masks of the following brain regions: dorsolateral prefrontal cortex (DLPFC), hippocampus, parahippocampal gyrus (PHG)

${ }^{1}$ Department of Human Genetics, NIMHANS, Hosur Road, Bangalore-560029, India

Full list of author information is available at the end of the article and posterior cingulate gyrus (PCG) [uncorrected $\mathrm{p}<0.01$; small-volume-correction for the respective mask (familywise-error) $\mathrm{p}<0.05$; 10-voxel threshold].

\section{Results}

There were no significant allele and genotype differences between patients and controls. However, a significant increase of heterozygous $(+14 \mathrm{bp} /-14 \mathrm{bp})$ genotype was observed in the female patients $(\mathrm{p} \leq 0.05)$. Interestingly, mother and the female patients also shared increased $+14 \mathrm{bp} /-14 \mathrm{bp}$ compatibility. Imaging analysis indicated that patients exhibiting $+14 \mathrm{bp} /-14 \mathrm{bp}$ genotypes had significantly deficient volume in the right hippocampus $[30,-13,-12]$ and right PHG $[41,-38,-11]$.

\section{Conclusion}

Our results demonstrate a possible role of HLA-G polymorphism and feto-maternal matching of HLA-G in conferring the risk of schizophrenia. Importantly, this genetic variant also influences brain morphometric measures. Taken together, these findings suggest HLA$\mathrm{G}$ could be an important biomarker for schizophrenia.

\section{Authors' details \\ 'Department of Human Genetics, NIMHANS, Hosur Road, Bangalore-560029, India. ${ }^{2}$ Translational Psychiatry Laboratory, Department of Psychiatry, Neurobiology Research Centre, NIMHANS, Hosur Road, Bangalore-560029, India.}

Published: 21 January 2014

doi:10.1186/1755-8166-7-S1-P43

Cite this article as: Rajasekaran et al:: Association of HLA-G 14bp INS/DEL

Polymorphism with brain morphology in Schizophrenia. Molecular Cytogenetics 2014 7(Suppl 1):P43. 